\title{
On the Intertwined Contributions of Physical and Digital Tools for the Teaching and Learning of Mathematics
}

\author{
Ricardo Nemirovsky ${ }^{1} \cdot$ Nathalie Sinclair $^{2,3}$
}

Published online: 1 July 2020

(C) Springer Nature Switzerland AG 2020

The idea of this special issue was born in a conversation between Sophie SouryLavergne and Ricardo Nemirovsky during the PME 42nd annual meeting in Umeå, Sweden. Sophie had been working on the notion of "duo of artefacts," combining tangible and digital tools for the learning and teaching of mathematics. Ricardo had a lifelong interest in practices of body motion for mathematics learning. They wondered about ways to enable the richness of digital environments without losing touch (pun intended) with the wide range of sensorial and kinesthetic abilities of all living beings. Manipulatives or physical objects, like ruler, compass, and paper folding are ingrained in the origins of mathematical ideas. Mathematics educators in all parts of the world have used them, as well as more pedagogically oriented objects, such as Cuisenaire rods, Montessori colour beads, abacuses, Dienes blocks, Geoboards and snap cubes, to immerse mathematical ideas in the feeling of their materiality. Meanwhile, the tantalizing possibilities opened up by the power and responsiveness of digital applications has generated enormous energy in our field towards experimenting with them. Hybrids digital/physical have also been developed, such as the Logo turtle as a robot, mousedragging in dynamic geometry, motion detectors, and the tagging of objects to be digitally recognized with radio-frequency identification (RFID).

Nevertheless, for some of us this amazing development left lingering concerns about whether something was being lost in the transition. Although imperfectly, we can distinguish digital tools as being computer-screen centred and tangible tools as being for holding, grabbing, and manual transport. In both cases: 1) the visual sense is or can be a central component of the learning experience and, 2) there is high potential for

Ricardo Nemirovsky

R.Nemirovsky@mmu.ac.uk

Nathalie Sinclair

nathalie_sinclair@sfu.ca

1 Manchester Metropolitan University, Manchester, UK

2 College of New Scholars, Royal Society of Canada, Ottawa, ON, Canada

3 Simon Fraser University, 8888 University Drive, Burnaby, BC V5A 1S6, Canada 
bodily engagement. These commonalities raise the questions that is the focus of this special issue: How do the learning affordances of digital and tangible tools differ from each other? In what cases they are or aren't mutually substitutable? Are there optimal combinations of digital and tangible tools? How do tangible and digital tools entangle differently with the aesthetic and affective dimensions of mathematics learning? Are there sequences for their alternate use that appear to enhance learning experiences? What theoretical frameworks can help us understand their differences and complementarities?

The goal of this special issue is the creation of a reference volume to foreground these research questions in the field. The call for papers has generated an enthusiastic response unexpectedly leading to the production of two issues. This is the first one. The articles in this first special issue range across mathematical topic and grade level, as well as across various digital and physical tools, some of which are familiar and some that are new.The opening essay, by Dr. Jeanne Bamberger, draws on threads from her research of decades ago and helps illustrate ways in which the intertwining of the physical and the digital has been around for a long time. We are pleased to see these threads being picked up anew in the articles that follow. We think that the number and quality of the submitted contributions attest to the relevance and urgency of these questions, emerging from the current evolution of research in mathematics education.

As co-editors of the two volumes, we wish to thank Sophie Soury-Lavergne for her work in framing the project and providing advice along the way. We'd also like to thank Richard Noss for his work in shepherding the special issue and providing highquality reviews on many of the articles. Finally, we thank all of the reviewers who provided critical and constructive suggestions.

Publisher's Note Springer Nature remains neutral with regard to jurisdictional claims in published maps and institutional affiliations. 